\title{
The Consistency Strength of Choiceless Failures of SCH *†
}

\author{
Arthur W. Apter \\ Department of Mathematics \\ Baruch College of CUNY \\ New York, New York 10010 USA \\ and \\ The CUNY Graduate Center, Mathematics \\ 365 Fifth Avenue \\ New York, New York 10016 USA \\ http://faculty.baruch.cuny.edu/apter \\ awapter@alum.mit.edu \\ Peter Koepke \\ Mathematisches Institut \\ Rheinische Friedrich-Wilhelms-Universität \\ Endenicher Allee 60 \\ D-53115 Bonn, Germany \\ http://www.math.uni-bonn.de/people/koepke \\ koepke@math.uni-bonn.de \\ May 31, 2009 \\ (revision as of September 16, 2009)
}

\begin{abstract}
We determine exact consistency strengths for various failures of the Singular Cardinals Hypothesis ( $\mathrm{SCH}$ ) in the setting of the Zermelo-Fraenkel axiom system ZF without the Axiom of Choice (AC). By the new notion of parallel Prikry forcing that we introduce, we obtain surjective failures of SCH using only one measurable cardinal, including a surjective failure of Shelah's pcf theorem about the size of the power set of $\aleph_{\omega}$. Using symmetric collapses to
\end{abstract}

*2000 Mathematics Subject Classifications: 03E25, 03E35, 03E45, 03E55.

$\dagger$ Keywords: Singular Cardinals Hypothesis (SCH), core model, parallel Prikry forcing, symmetric inner model.

$\ddagger$ The first author's research was partially supported by PSC-CUNY grants, CUNY Collaborative Incentive grants, and DFG-NWO Bilateral Grant KO 1353-5/1/DN 62-630. In addition, the first author wishes to thank the members of the mathematical logic group in Bonn for all of the hospitality shown him during his spring 2009 Scholar Incentive Leave visit to the Mathematisches Institut. 
$\aleph_{\omega}, \aleph_{\omega_{1}}$, or $\aleph_{\omega_{2}}$, we show that injective failures at $\aleph_{\omega}$, $\aleph_{\omega_{1}}$, or $\aleph_{\omega_{2}}$ can have relatively mild consistency strengths in terms of Mitchell orders of measurable cardinals. Injective failures of both the aforementioned theorem of Shelah and Silver's theorem that GCH cannot first fail at a singular strong limit cardinal of uncountable cofinality are also obtained. Lower bounds are shown by core model techniques and methods due to Gitik and Mitchell.

\section{Introduction}

One of the first applications of Paul Cohen's method of forcing was given by Easton [5] (see also [14]), who showed that for regular cardinals $\kappa$, the value of the continuum function $2^{\kappa}$ is largely undetermined by the standard ZFC axioms of set theory. To extend those investigations to singular cardinals $\kappa$ proved significantly more difficult and led to the formulation of the Singular Cardinals Hypothesis (SCH):

$$
(\operatorname{cof}(\kappa)<\kappa) \wedge(\forall \nu<\kappa)\left[2^{\nu}<\kappa\right] \Longrightarrow \kappa^{\operatorname{cof}(\kappa)}=\kappa^{+}
$$

Using the Axiom of Choice (AC), the $\mathrm{SCH}$ readily implies that

$$
(\operatorname{cof}(\kappa)<\kappa) \wedge(\forall \nu<\kappa)\left[2^{\nu}<\kappa\right] \Longrightarrow 2^{\kappa}=\kappa^{+}
$$

i.e., the continuum function at $\kappa$ takes the smallest possible value.

The Singular Cardinals Hypothesis had a decisive impact on the further development of axiomatic set theory, leading to sophisticated methods in combinatorics, forcing, and the theory of inner models. Jack Silver (see [18] and [14]) proved some instances of SCH from the ZFC axioms and forced violations of SCH in other cases. Ronald Jensen showed (see [2]) that violating the SCH requires the existence of large cardinals in inner models of set theory. Saharon Shelah's pcf theory [17] extends Silver's analysis also to the case of countable cofinality. Moti Gitik [9], [11] determined the exact consistency strength of the negation of $\mathrm{SCH}$, in the presence of $\mathrm{AC}$, to be

$$
\mathrm{Con}(\mathrm{ZFC}+\neg \mathrm{SCH}) \Longleftrightarrow \mathrm{Con}\left(\mathrm{ZFC}+\exists \kappa\left[o(\kappa)=\kappa^{++}\right]\right)
$$

Note that the work of [9] uses (previously) unpublished ideas of Woodin.

The results and arguments mentioned so far essentially involve the Axiom of Choice. In this paper, we examine the status of $\neg \mathrm{SCH}$ without $\mathrm{AC}$. We will obtain equiconsistencies for three 
of our main theorems, and upper and lower bounds in consistency strength for our fourth main theorem. There will be sharp differences between the non-AC and AC situations, as we shall explain shortly.

The logical negation of the $\mathrm{SCH}$ at $\kappa$ reads

$$
(\operatorname{cof}(\kappa)<\kappa) \wedge(\forall \nu<\kappa)\left[2^{\nu}<\kappa\right] \wedge\left(\kappa^{\operatorname{cof}(\kappa)} \neq \kappa^{+}\right)
$$

Without $\mathrm{AC}, \kappa^{\operatorname{cof}(\kappa)} \neq \kappa^{+}$does not imply that $\kappa^{\operatorname{cof}(\kappa)}$ is larger than $\kappa^{+}$. So we have to express largeness in terms of cardinality theory without AC. In the sequel, we shall distinguish between surjective failures of SCH, e.g.,

$$
(\operatorname{cof}(\kappa)<\kappa) \wedge(\forall \nu<\kappa)\left[2^{\nu}<\kappa\right] \wedge\left(\text { There is a surjective } f:[\kappa]^{\operatorname{cof}(\kappa)} \rightarrow \kappa^{++}\right)
$$

and injective failures, e.g.,

$$
(\operatorname{cof}(\kappa)<\kappa) \wedge(\forall \nu<\kappa)\left[2^{\nu}<\kappa\right] \wedge\left(\text { There is an injective } f: \kappa^{++} \rightarrow[\kappa]^{\operatorname{cof}(\kappa)}\right)
$$

Note that $2^{\nu}<\kappa$ for $\nu<\kappa$ implies that $\wp(\nu)$ is well-orderable in some order type less than $\kappa$.

We prove that surjective failures of $\mathrm{SCH}$ in $\mathrm{ZF}+\neg \mathrm{AC}$ are of mild consistency strength, i.e., only one measurable cardinal, and that a surjective failure at minimal singular cardinals of cofinality $\omega$ like $\aleph_{\omega}$ does not raise the strength. These surjective failures at $\aleph_{\omega}$ may be beyond what is currently known to be possible in ZFC, or even contradict what is possible in ZFC. We further construct injective failures of $\mathrm{SCH}$ at $\aleph_{\omega}$ that are beyond what is currently known to be possible in ZFC, or even contradict what is possible in ZFC, and show that they have fairly mild consistency strengths as well. We in addition force injective failures of SCH at $\aleph_{\omega_{1}}$ and $\aleph_{\omega_{2}}$ that are impossible in ZFC, and demonstrate that their consistency strengths are also quite innocuous. Specifically, we prove the following theorems, emphasizing that throughout, whenever we talk about GCH holding below a cardinal $\kappa$, we literally mean the same thing as when $\mathrm{AC}$ is true, i.e., that for every (well-ordered) cardinal $\nu<\kappa, \wp(\nu)$ is well-orderable and has cardinality $\nu^{+}$.

\section{Theorem 1}


For a fixed $\alpha \geq 2$, the following theories are equiconsistent:

$$
Z F C+\exists \kappa[\kappa \text { is measurable }]
$$

and

$$
Z F+\neg A C+G C H \text { holds below } \aleph_{\omega}+\text { There is a surjective } f:\left[\aleph_{\omega}\right]^{\omega} \rightarrow \aleph_{\omega+\alpha} .
$$

\section{Theorem 2}

For a fixed $n<\omega, n \geq 1$, the following theories are equiconsistent:

$$
Z F C+\exists \kappa\left[(\operatorname{cof}(\kappa)=\omega) \wedge(\forall i<\omega)(\forall \lambda<\kappa)(\exists \delta<\kappa)\left[(\delta>\lambda) \wedge\left(o(\delta) \geq \delta^{+i}\right)\right]\right]
$$

and

$$
Z F+\neg A C+G C H \text { holds below } \aleph_{\omega}+\text { There is an injective } f: \aleph_{\omega_{n}} \rightarrow\left[\aleph_{\omega}\right]^{\omega} \text {. }
$$

When talking about a choiceless injective failure of SCH for singular cardinals of uncountable cofinality, the situation is somewhat different. More explicitly, we have the following two theorems, which we state in a very specific, concrete manner for the sake of exposition. There are two distinct cases, depending on whether the singular cardinal has cofinality at least $\omega_{2}$ or cofinality $\omega_{1}$. We shall use $\aleph_{\omega_{2}}$ and $\aleph_{\omega_{1}}$ as our prototypes. The reason for the split in cases, as well as the many additional possibilities, will be discussed in Section 8 .

\section{Theorem 3}

The following theories are equiconsistent:

$$
Z F C+\exists \kappa\left[o(\kappa)=\kappa^{++}+\omega_{2}\right]
$$

and

$$
Z F+\neg A C+G C H \text { holds below } \aleph_{\omega_{2}}+\text { There is an injective } f: \aleph_{\omega_{2}+2} \rightarrow\left[\aleph_{\omega_{2}}\right]^{\omega_{2}} \text {. }
$$

\section{Theorem 4}


a) If the theory

$$
Z F C+\exists \kappa\left[o(\kappa)=\kappa^{++}+\omega_{1}\right]
$$

is consistent, then so is the theory

$$
Z F+\neg A C+G C H \text { holds below } \aleph_{\omega_{1}}+\text { There is an injective } f: \aleph_{\omega_{1}+2} \rightarrow\left[\aleph_{\omega_{1}}\right]^{\omega_{1}} \text {. }
$$

b) If the theory

$$
Z F+\neg A C+G C H \text { holds below } \aleph_{\omega_{1}}+\text { There is an injective } f: \aleph_{\omega_{1}+2} \rightarrow\left[\aleph_{\omega_{1}}\right]^{\omega_{1}}
$$

is consistent, then so is the theory

$$
Z F C+\exists \kappa\left[o(\kappa)=\kappa^{++}\right]
$$

In Theorems $2-4$, for $\lambda=\aleph_{\omega}, \lambda=\aleph_{\omega_{1}}$, or $\lambda=\aleph_{\omega_{2}}$, our proofs will show that the injection into $[\lambda]^{\operatorname{cof}(\lambda)}$ can be safely replaced with an injection into $\wp(\lambda)$. As we shall discuss later, the analogous fact for Theorem 1 may not hold, i.e., we have not been able to replace the surjection from $\left[\aleph_{\omega}\right]^{\omega}$ onto $\aleph_{\omega+\alpha}$ with a surjection from $\wp\left(\aleph_{\omega}\right)$ onto $\aleph_{\omega+\alpha}$ and obtain the lower bound in consistency strength of one measurable cardinal. Also, to avoid trivialities, the surjection in Theorem 1 is onto a cardinal greater than or equal to $\aleph_{\omega+2}$, and the injection in Theorems 3 and 4 is from a cardinal of size at least $\lambda^{++}$.

Loosely speaking, the cardinalities of $\left[\aleph_{\omega}\right]^{\omega}$ and $\left[\aleph_{\omega_{1}}\right]^{\omega_{1}}$ in these situations may be blown up so that they "contradict" the conclusions of the seminal theorems of Silver [18] and Shelah [17] in surjective and injective ways. In particular, Theorems 3 and 4 provide an injection from $\lambda^{++}$into the power set of a singular cardinal $\lambda$ of uncountable cofinality, together with GCH holding below $\lambda$. This, of course, is in sharp contrast to Silver's ZFC result [18] that GCH cannot first fail at a singular strong limit cardinal of uncountable cofinality.

In addition, Theorem 1 yields that it is possible to have a surjection from $\wp\left(\aleph_{\omega}\right)$ onto any $\aleph_{\beta}$ together with GCH holding below $\aleph_{\omega}$, and Theorem 2 tells us that it is possible to have an injection from $\aleph_{\omega_{n}}$ into $\wp\left(\aleph_{\omega}\right)$ for any $n \geq 1, n<\omega$ together with GCH holding below $\aleph_{\omega}$. We now compare 
this with the situation in ZFC. Although it is known (see [8]) how to force SCH to fail at $\aleph_{\omega}$ with the size of the power set of $\aleph_{\omega}$ arbitrarily large below $\aleph_{\omega_{1}}$, it is currently unknown (see [8] for a discussion) whether it is possible to force SCH to fail at $\aleph_{\omega}$ with $2^{\aleph_{\omega}} \geq \aleph_{\omega_{1}}$. It is known, however, that a failure of SCH at $\aleph_{\omega}$ in conjunction with $2^{\aleph_{\omega}} \geq \aleph_{\omega_{1}}$ is very strong, and in fact yields the existence of an inner model containing a Woodin cardinal (see [13]). When $n \geq 4$, we get choiceless "counterexamples" to Shelah's theorem [17] that when $\aleph_{\omega}$ is a strong limit cardinal, $2^{\aleph_{\omega}}<\aleph_{\omega_{4}}$. All of this is once again in sharp contrast to the situation in ZFC.

A crucial feature of the symmetric submodels of the parallel Prikry forcing or the symmetric collapses will be that they can be approximated from within by certain submodels in which AC holds. The lower bound on consistency strength of one measurable cardinal for surjective failures of SCH will consequently be determined using the Dodd-Jensen core model [3], [4] for sequences of measures. Since core model theory uses the Axiom of Choice, we employ HOD-like inner models. Lower bounds on consistency strength for injective failures of SCH will be obtained by using Gitik and Mitchell's work [12] (which is also discussed in [10]).

This paper is structured as follows. In Section 2, we introduce our new notion of parallel Prikry forcing, which will be used to construct choiceless, surjective failures of SCH. We demonstrate some of its basic properties, and also produce a choiceless, symmetric submodel $N$ of a generic extension via parallel Prikry forcing. In Section 3, we prove the lemmas necessary to provide a detailed analysis of $N$. In Section 4, we show how the appropriate construction of $N$ may be used to obtain these surjective failures of SCH at some previously measurable cardinal $\kappa$ which now has cofinality $\omega$. In Section 5, we give a general paradigm for symmetrically collapsing large cardinals at which $\mathrm{SCH}$ fails, either in a model of ZFC or surjectively in a choiceless model of ZF, down to small singular limit cardinals, such as $\aleph_{\omega}$, $\aleph_{\omega_{1}}$, or $\aleph_{\omega_{2}}$. In Section 6 , we establish the upper bounds in consistency strength of our main theorems via forcing, thereby providing the models in which SCH will fail either surjectively or injectively at $\aleph_{\omega}, \aleph_{\omega_{1}}$, or $\aleph_{\omega_{2}}$. In Section 7 , we establish the lower bounds in consistency strength of our main theorems via techniques from inner model theory, which completes the proofs of these theorems. Section 8 contains our concluding remarks, 
along with a discussion of some generalizations of the main theorems provable by our methods.

\section{$2 \quad$ Parallel Prikry forcing}

Parallel Prikry forcing is a subforcing of a finite support product of Prikry forcings, where the Prikry sequences formed are eventually interlaced in a very systematic fashion. This prevents the coding of unwanted information into the generic extension.

Fix a measurable cardinal $\kappa$ and a normal measure $\mathcal{U}$ on $\kappa$. Fix in addition a set $Z \subseteq$ Ord, which will be the support of the subsequent forcing.

We will now define $Z$-fold parallel Prikry forcing for the measure $\mathcal{U}{ }^{1}$ We will occasionally write $\left(\mathbb{P}_{Z}, \leq\right)$, although more often, we will write $(\mathbb{P}, \leq)$ instead of $\left(\mathbb{P}_{Z}, \leq\right)$ for simplicity. Getting specific, a sequence $p=\left(s_{\alpha}, A_{\alpha}\right)_{\alpha \in Z}$ is a condition in $\mathbb{P}$ iff

1. $\forall \alpha \in Z\left[\left(s_{\alpha} \in[\kappa]^{<\omega}\right) \wedge\left(A_{\alpha} \in \mathcal{U}\right) \wedge\left(\max \left(s_{\alpha}\right)<\min \left(A_{\alpha}\right)\right)\right]$; here, we set $\max (\emptyset)=-1$.

2. $\operatorname{dom}(p):=\left\{\alpha \in Z \mid A_{\alpha} \neq \kappa\right\}$ is finite.

We write $\left(s_{\alpha}, A_{\alpha}\right)$ instead of $\left(s_{\alpha}, A_{\alpha}\right)_{\alpha \in Z}$. We will, however, occasionally abuse notation and also use $\left(s_{\alpha}, A_{\alpha}\right)$ to mean an individual component of $\left(s_{\alpha}, A_{\alpha}\right)_{\alpha \in Z}$. Conditions $p^{\prime}=\left(s_{\alpha}^{\prime}, A_{\alpha}^{\prime}\right)$ and $p=\left(s_{\alpha}, A_{\alpha}\right)$ in $\mathbb{P}$ are partially ordered by $p^{\prime} \leq p$ (i.e., $p^{\prime}$ is stronger than $p$ ) iff there is an integer $n<\omega$ such that

1. $\forall \alpha \in \operatorname{dom}(p)\left[\left(\operatorname{otp}\left(s_{\alpha}^{\prime} \backslash s_{\alpha}\right)=n\right) \wedge\left(s_{\alpha}^{\prime} \backslash s_{\alpha} \subseteq A_{\alpha}\right)\right]$.

2. $(\forall \alpha, \beta \in \operatorname{dom}(p))\left(\forall \xi \in s_{\alpha}^{\prime} \backslash s_{\alpha}\right)\left(\forall \zeta \in s_{\beta}\right)[\xi>\zeta]$.

3. $(\forall \alpha<\beta \in \operatorname{dom}(p))(\forall i<n)\left[\left(s_{\alpha}^{\prime} \backslash s_{\alpha}\right)[i]<\left(s_{\beta}^{\prime} \backslash s_{\beta}\right)[i]\right]$, where $s[i]$ denotes the $i$-th element of the monotone enumeration of the set $s$ of ordinals.

4. $(\forall \alpha, \beta \in \operatorname{dom}(p))(\forall i<n)\left[(i+1<n) \Longrightarrow\left(\left(s_{\alpha}^{\prime} \backslash s_{\alpha}\right)[i]<\left(s_{\beta}^{\prime} \backslash s_{\beta}\right)[i+1]\right)\right]$.

5. $\forall \alpha \in \operatorname{dom}(p)\left[A_{\alpha}^{\prime} \subseteq A_{\alpha}\right]$.

\footnotetext{
${ }^{1}$ Parallel Prikry forcing was first defined using a sequence of pairwise distinct normal measures on $\kappa$. We are indebted to Gunter Fuchs, who pointed out that a single normal measure suffices.
} 
Intuitively, $p^{\prime}=\left(s_{\alpha}^{\prime}, A_{\alpha}^{\prime}\right) \leq p=\left(s_{\alpha}, A_{\alpha}\right)$ means that on the domain of $p$, the following hold:

1. The stems $s_{\alpha}$ are extended into the corresponding reservoir sets $A_{\alpha}$ in a systematic fashion.

2. The extension points are chosen greater than all of the previous stem points.

3. There are the same number of new points at all indices in $\operatorname{dom}(p)$, and these are chosen in layers which are strictly ascending.

Moreover, reservoirs may be thinned out, and new stems outside the old domain may be grown. The following diagram indicates how $p^{\prime}$ (with grey infill color) extends $p$ (with thick black contours).

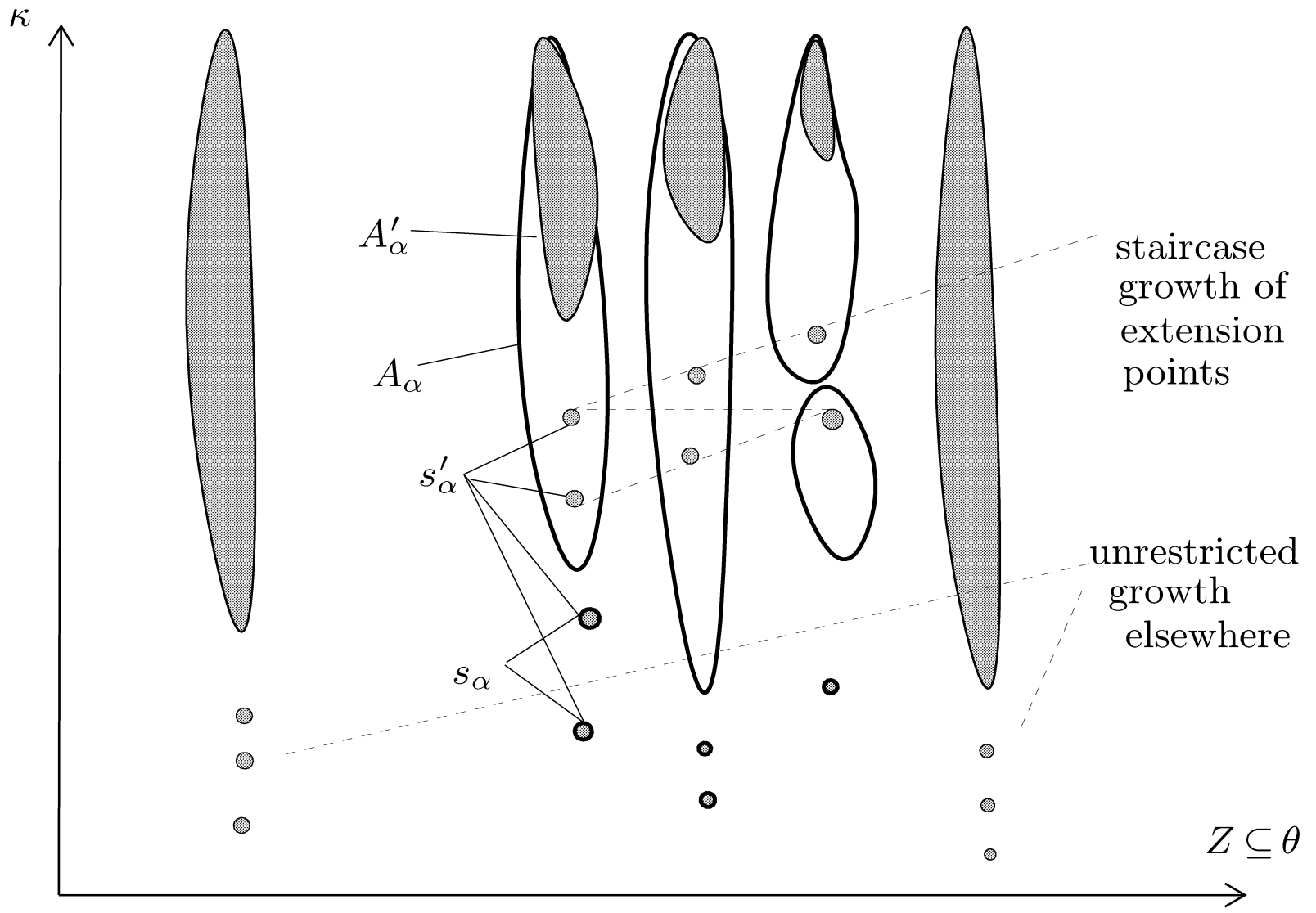

Let $G$ be $\mathbb{P}$-generic over $V$. $G$ adjoins a system $\left(C_{\alpha} \mid \alpha \in Z\right)$, where for a fixed $\alpha$,

$$
C_{\alpha}=\bigcup\left\{s_{\alpha} \mid\left(s_{\beta}, A_{\beta}\right)_{\beta \in Z} \in G\right\} .
$$

Density arguments show that the $C_{\alpha}$ are distinct. Lemma 1 shows that the $C_{\alpha}$ are Prikry sequences for the measure $\mathcal{U}$. 


\section{Lemma 1}

a) Let $\gamma \in Z$. Then $C_{\gamma}$ is a Prikry sequence for $\mathcal{U}$, i.e.,

$$
\forall X \in \wp(\kappa) \cap V\left[(X \in \mathcal{U}) \Longleftrightarrow\left(C_{\gamma} \backslash X \text { is finite }\right)\right]
$$

This implies that $C_{\gamma}$ is cofinal in $\kappa$ of order type $\omega$.

b) Let $\gamma, \delta \in Z, \gamma<\delta$. Then $C_{\gamma} \cap C_{\delta}$ is finite, and therefore $C_{\gamma} \Delta C_{\delta}$ is infinite.

\section{Proof}

a) Let $X \in \wp(\kappa) \cap V$. Assume that $X \in \mathcal{U}$. Take $p=\left(s_{\alpha}, A_{\alpha}\right) \in G$ such that $A_{\gamma} \subseteq X$. By the definition of $C_{\gamma}, C_{\gamma} \backslash s_{\gamma} \subseteq A_{\gamma} \subseteq X$. Hence, $C_{\gamma} \backslash X \subseteq s_{\gamma}$ is finite.

For the converse, assume that $X \notin \mathcal{U}$. We show that $C_{\gamma} \backslash X$ is cofinal in $\kappa$. Let $\nu<\kappa$. Take $p=\left(s_{\alpha}, A_{\alpha}\right) \in G$ such that $A_{\gamma} \subseteq(\kappa \backslash X) \cap(\kappa \backslash \nu)$. By the definition of $C_{\gamma}$,

$$
C_{\gamma} \backslash s_{\gamma} \subseteq A_{\gamma} \subseteq(\kappa \backslash X) \cap(\kappa \backslash \nu)
$$

and by density, $C_{\gamma} \backslash s_{\gamma} \neq \emptyset$. Say $\xi \in C_{\gamma} \backslash s_{\gamma}$. Then $\xi \in C_{\gamma} \backslash X$ and $\xi \geq \nu$, as required.

b) Take $p=\left(s_{\alpha}, A_{\alpha}\right) \in G$ such that $\gamma, \delta \in \operatorname{dom}(p), \gamma<\delta$. It suffices to show that $C_{\gamma} \cap C_{\delta} \subseteq$ $s_{\gamma} \cap s_{\delta}$. Consider $\xi \in C_{\gamma} \cap C_{\delta}$. Take $p^{\prime}=\left(s_{\alpha}^{\prime}, A_{\alpha}^{\prime}\right) \in G$ such that $p^{\prime} \leq p$ and $\xi \in s_{\gamma}^{\prime} \cap s_{\delta}^{\prime}$. By requirement (3) of the definition of $\leq,\left(s_{\gamma}^{\prime} \backslash s_{\gamma}\right) \cap s_{\delta}^{\prime}=\emptyset$ and $\left(s_{\delta}^{\prime} \backslash s_{\delta}\right) \cap s_{\gamma}^{\prime}=\emptyset$. This implies that $\xi \in s_{\gamma} \cap s_{\delta}$

Lemma $2(\mathbb{P}, \leq)$ satisfies the $\kappa^{+}$-chain condition.

Proof Let $\left\{\left(s_{\alpha}^{i}, A_{\alpha}^{i}\right) \mid i<\kappa^{+}\right\} \subseteq \mathbb{P}$. We want to show that at least two of the $\left(s_{\alpha}^{i}, A_{\alpha}^{i}\right)$ are compatible in $\mathbb{P}$. By a $\Delta$-system argument, we may assume that the domains $\operatorname{dom}\left(\left(s_{\alpha}^{i}, A_{\alpha}^{i}\right)\right)$ form a $\Delta$-system with kernel $Z_{0} \in[Z]^{<\omega}$. By a pigeonhole argument, we may assume that there are $i<j<\kappa^{+}$such that $\left(s_{\alpha}^{i}\right)_{\alpha \in Z_{0}}=\left(s_{\alpha}^{j}\right)_{\alpha \in Z_{0}}$. Then $\left(s_{\alpha}^{i}, A_{\alpha}^{i}\right)$ and $\left(s_{\alpha}^{j}, A_{\alpha}^{j}\right)$ have a common refinement $\left(t_{\alpha}, B_{\alpha}\right)$ defined by

$$
t_{\alpha}=\left\{\begin{array}{l}
s_{\alpha}^{i}, \text { if } \alpha \in \operatorname{dom}\left(\left(s_{\alpha}^{i}, A_{\alpha}^{i}\right)\right) \\
s_{\alpha}^{j}, \text { if } \alpha \in \operatorname{dom}\left(\left(s_{\alpha}^{j}, A_{\alpha}^{j}\right)\right) \\
\emptyset, \text { otherwise }
\end{array}\right.
$$


and

$$
B_{\alpha}=A_{\alpha}^{i} \cap A_{\alpha}^{j}
$$

So forcing with $\mathbb{P}$ preserves all cardinals greater than or equal to $\kappa^{+}$. On the other hand, in case $Z$ is infinite, the measurable cardinal $\kappa$ is made countable. To see this, let $\left(C_{\alpha}\right)_{\alpha \in Z}$ be the system of Prikry sequences added by the forcing. Then a simple density argument shows that the function

$$
\alpha \mapsto \min \left(C_{\alpha}\right)
$$

maps any countable subset of $Z$ onto $\kappa$.

Therefore, we shall work instead with the symmetric submodel

$$
N=\operatorname{HOD}^{V[G]}\left(\bigcup_{\alpha \in Z} \tilde{C}_{\alpha} \cup\left\{\left(\tilde{C}_{\alpha} \mid \alpha \in Z\right)\right\}\right),
$$

where $\tilde{C}_{\alpha}=\left\{C \in \wp(\kappa) \mid C \Delta C_{\alpha}\right.$ is finite $\}$. This is the class of sets which are hereditarily definable in the generic extension from finitely many parameters from the class $\operatorname{Ord} \cup\left\{C_{\alpha} \mid \alpha \in Z\right\} \cup\left\{\left(\tilde{C}_{\alpha} \mid\right.\right.$ $\alpha \in Z)\}$. If, e.g., $Z=\kappa^{++}$, as we shall show in Section 4 , the following will give a surjection contradicting $\mathrm{SCH}$.

Lemma 3 In $N$, there is a surjection $f:[\kappa]^{\omega} \rightarrow Z$.

Proof Define $f$ using the parameter $\left(\tilde{C}_{\alpha} \mid \alpha \in Z\right)$ by

$$
X \mapsto\left\{\begin{array}{l}
\text { The unique } \alpha \in Z \text { such that } X \in \tilde{C}_{\alpha}, \text { if that exists, } \\
0, \text { otherwise. }
\end{array}\right.
$$

It follows that $f$ is surjective, since $f\left(C_{\alpha}\right)=\alpha$ by Lemma 1a).

\section{$3 \quad$ Finite support approximations}

The model $N$ will be analyzed using finite support approximations. We show that parallel Prikry forcing with a finite support $Z \subseteq$ Ord is equivalent to standard Prikry forcing. Note that standard Prikry forcing corresponds to the forcing $\mathbb{P}_{1}=\mathbb{P}_{\{0\}}$. For simplicity, we consider sets $Z=\ell$, where $\ell<\omega$. 


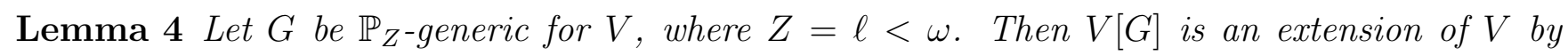
Prikry forcing $\mathbb{P}_{1}$. Therefore, by the properties of standard Prikry forcing, $V[G]$ has the same bounded subsets as $V$.

Proof Choose a condition $p=\left(s_{i}, A\right) \in G$ such that $\operatorname{dom}(p)=Z$. Note that we may densely assume that the finitely many $A_{i}$ are all equal to $A$, and that $A \neq \kappa$. Then $V[G]$ is a generic extension of $V$ by the restricted partial ordering $\mathbb{P}_{Z}^{\prime}=\left\{q \in \mathbb{P}_{Z} \mid q \leq p\right\}$. Observing that $(\emptyset, A) \in \mathbb{P}_{1}$, define $\mathbb{P}_{1}^{\prime}=\left\{r \in \mathbb{P}_{1} \mid r \leq(\emptyset, A)\right\}$. It suffices to define a dense embedding $\pi$ from $\mathbb{P}_{Z}^{\prime}$ into $\mathbb{P}_{1}^{\prime}$.

Consider $\left(s_{i}^{\prime}, B_{i}\right) \in \mathbb{P}_{Z}^{\prime}$. For $i \in Z$, let

$$
s_{i}^{\prime} \backslash s_{i}=\left\{\xi_{i}^{0}, \xi_{i}^{1}, \ldots, \xi_{i}^{n-1}\right\}
$$

where

$$
\xi_{0}^{0}<\xi_{1}^{0}<\cdots<\xi_{\ell-1}^{0}<\xi_{0}^{1}<\xi_{1}^{1}<\cdots<\xi_{\ell-1}^{1}<\cdots<\xi_{0}^{n-1}<\xi_{1}^{n-1}<\cdots<\xi_{\ell-1}^{n-1}
$$

Then let $\pi\left(\left(s_{i}^{\prime}, B_{i}\right)\right)=\left(t, \bigcap_{i<\ell} B_{i}\right)$, where

$$
t=\left\{\xi_{0}^{0}, \xi_{1}^{0}, \ldots, \xi_{\ell-1}^{0}, \xi_{0}^{1}, \xi_{1}^{1}, \ldots, \xi_{\ell-1}^{1}, \ldots, \xi_{0}^{n-1}, \xi_{1}^{n-1}, \ldots, \xi_{\ell-1}^{n-1}\right\}
$$

This obviously defines a dense embedding.

Let us again consider an arbitrary support $Z \subseteq$ Ord and a finite subset $Z_{0} \subseteq Z$. We define restrictions to $Z_{0}$ by

$$
\mathbb{P} \uparrow Z_{0}=\left\{p \uparrow Z_{0} \mid p \in \mathbb{P}\right\}
$$

and

$$
G \uparrow Z_{0}=\left\{p \uparrow Z_{0} \mid p \in G\right\}
$$

Lemma 5 Let $G$ be $\mathbb{P}$-generic. Then $G \uparrow Z_{0}$ is $\mathbb{P} \uparrow Z_{0}$-generic.

\section{Proof Easy.}

The approximation of the model $\operatorname{HOD}^{V[G]}\left(\bigcup_{\alpha \in Z} \tilde{C}_{\alpha} \cup\left\{\left(\tilde{C}_{\alpha} \mid \alpha \in Z\right)\right\}\right)$ by finite support parallel Prikry extensions will be based on certain symmetries of the partial ordering $(\mathbb{P}, \leq)$. 
Lemma 6 Let $p=\left(s_{\alpha}, A_{\alpha}\right) \in \mathbb{P}$. Set $p^{-}=\left(\emptyset, A_{\alpha}\right) \in \mathbb{P}, \mathbb{P}_{p}=\{q \in \mathbb{P} \mid q \leq p\}$, and $\mathbb{P}_{p^{-}}=\{q \in \mathbb{P} \mid$ $\left.q \leq p^{-}\right\}$. Then the following hold:

a) The map

$$
\pi:\left(t_{\alpha}, B_{\alpha}\right) \mapsto\left(t_{\alpha} \backslash s_{\alpha}, B_{\alpha}\right)
$$

is an order isomorphism between $\left(\mathbb{P}_{p}, \leq\right)$ and $\left(\mathbb{P}_{p^{-}}, \leq\right)$.

b) $D$ is dense in $\mathbb{P}$ below $p^{-}$iff $\pi^{-1}[D]$ is dense in $\mathbb{P}$ below $p$.

c) $H$ is $\mathbb{P}$-generic below $p^{-}$iff $\pi^{-1}[H]$ is $\mathbb{P}$-generic below $p$.

d) If $H$ is $\mathbb{P}$-generic below $p^{-}$, then for every sequence $\left(s_{\alpha}^{\prime}\right)_{\alpha \in Z}$ with $\max \left(s_{\alpha}^{\prime}\right)<\min \left(A_{\alpha}\right)$, the set

$$
\left\{\left(s_{\alpha}^{\prime} \cup u_{\alpha}, B_{\alpha}\right) \mid\left(u_{\alpha}, B_{\alpha}\right) \in H\right\}
$$

is $\mathbb{P}$-generic with $\left(s_{\alpha}^{\prime}, A_{\alpha}\right) \in\left\{\left(s_{\alpha}^{\prime} \cup u_{\alpha}, B_{\alpha}\right) \mid\left(u_{\alpha}, B_{\alpha}\right) \in H\right\}$.

Proof Obviously, the map having domain $\mathbb{P}_{p^{-}}$defined by

$$
\left(u_{\alpha}, B_{\alpha}\right) \mapsto\left(s_{\alpha} \cup u_{\alpha}, B_{\alpha}\right)
$$

is the inverse of $\pi$, and hence $\pi$ is a bijection. The definition of the order relation $\leq$ implies immediately that $\pi$ is order preserving. Then $\pi$ and $\pi^{-1}$ preserve density and genericity. Property d) follows directly from c).

Let $\dot{C}_{\alpha}$ be a canonical name for the $\alpha$-th Prikry sequence added by forcing with $\mathbb{P}$, and let $\dot{D}$ be a canonical name for the sequence $\left(\tilde{C}_{\alpha} \mid \alpha \in Z\right)$ used in the definition of $N$.

Lemma 7 Let $\varphi$ be an $\in$-formula and $\varphi\left(\vec{\xi}, \dot{C}_{\alpha_{0}}, \ldots, \dot{C}_{\alpha_{n-1}}, \dot{D}\right)$ be a forcing sentence. Let $p=$ $\left(s_{\alpha}, A_{\alpha}\right) \in \mathbb{P}, q=\left(t_{\alpha}, B_{\alpha}\right) \in \mathbb{P}$ be such that $p \nmid\left\{\alpha_{0}, \ldots, \alpha_{n-1}\right\}=q \uparrow\left\{\alpha_{0}, \ldots, \alpha_{n-1}\right\}$. Then we cannot have that $p \Vdash \varphi\left(\vec{\xi}, \dot{C}_{\alpha_{0}}, \ldots, \dot{C}_{\alpha_{n-1}}, \dot{D}\right)$ and $q \Vdash \neg \varphi\left(\vec{\xi}, \dot{C}_{\alpha_{0}}, \ldots, \dot{C}_{\alpha_{n-1}}, \dot{D}\right)$ simultaneously.

Proof Assume towards a contradiction that $p \Vdash \varphi\left(\overrightarrow{\check{\xi}}, \dot{C}_{\alpha_{0}}, \ldots, \dot{C}_{\alpha_{n-1}}, \dot{D}\right)$ and $q \Vdash \neg \varphi\left(\vec{\xi}, \dot{C}_{\alpha_{0}}, \ldots, \dot{C}_{\alpha_{n-1}}, \dot{D}\right)$. Set $r^{-}=\left(\emptyset, A_{\alpha} \cap B_{\alpha}\right)$. Take a set $H$ which is $\mathbb{P}$-generic below $r^{-}$. 
Set

$$
H_{p}=\left\{\left(s_{\alpha} \cup u_{\alpha}, E_{\alpha}\right) \mid\left(u_{\alpha}, E_{\alpha}\right) \in H\right\} \text { and } H_{q}=\left\{\left(t_{\alpha} \cup u_{\alpha}, E_{\alpha}\right) \mid\left(u_{\alpha}, E_{\alpha}\right) \in H\right\} \text {. }
$$

By Lemma $6, H_{p}$ and $H_{q}$ are $\mathbb{P}$-generic, with $p \in H_{p}$ and $q \in H_{q}$ respectively. Hence,

$$
V\left[H_{p}\right] \vDash \varphi\left(\vec{\xi},\left(\dot{C}_{\alpha_{0}}\right)^{H_{p}}, \ldots,\left(\dot{C}_{\alpha_{n-1}}\right)^{H_{p}}, \dot{D}^{H_{p}}\right) \text { and } V\left[H_{q}\right] \vDash \neg \varphi\left(\vec{\xi},\left(\dot{C}_{\alpha_{0}}\right)^{H_{q}}, \ldots,\left(\dot{C}_{\alpha_{n-1}}\right)^{H_{q}}, \dot{D}^{H_{q}}\right)
$$

For $i<n, s_{\alpha_{i}}=t_{\alpha_{i}}$ and $\left(\dot{C}_{\alpha_{i}}\right)^{H_{p}}=\left(\dot{C}_{\alpha_{i}}\right)^{H_{q}}$. For $\alpha \notin\left\{\alpha_{0}, \ldots, \alpha_{n-1}\right\},\left(\dot{C}_{\alpha}\right)^{H_{p}} \Delta\left(\dot{C}_{\alpha}\right)^{H_{q}} \subseteq s_{\alpha} \cup t_{\alpha}$ is finite. Thus, $\dot{D}^{H_{p}}=\dot{D}^{H_{q}}$. Since the generic sets $H, H_{p}$, and $H_{q}$ differ only by finite sets in $V$, $V[H]=V\left[H_{p}\right]=V\left[H_{q}\right]$. Then (1) leads to the contradiction

$$
V[H] \vDash \varphi\left(\vec{\xi},\left(\dot{C}_{\alpha_{0}}\right)^{H_{p}}, \ldots,\left(\dot{C}_{\alpha_{n-1}}\right)^{H_{p}}, \dot{D}^{H_{p}}\right) \text { and } V[H] \vDash \neg \varphi\left(\vec{\xi},\left(\dot{C}_{\alpha_{0}}\right)^{H_{p}}, \ldots,\left(\dot{C}_{\alpha_{n-1}}\right)^{H_{p}}, \dot{D}^{H_{p}}\right)
$$

Lemma 8 Let $G$ be $\mathbb{P}$-generic, with $C_{\alpha}=\left(\dot{C}_{\alpha}\right)^{G}$ for $\alpha \in Z$ and $D=\dot{D}^{G}$. Let $X \in V[G]$ be defined by

$$
X=\left\{\zeta \in \operatorname{Ord} \mid V[G] \vDash \varphi\left(\zeta, \vec{\xi}, C_{\alpha_{0}}, \ldots, C_{\alpha_{n-1}}, D\right)\right\}
$$

where $\alpha_{0}, \ldots, \alpha_{n-1} \in Z$. Then $X \in V\left[G \uparrow\left\{\alpha_{0}, \ldots, \alpha_{n-1}\right\}\right]$.

Proof Let $Z_{0}=\left\{\alpha_{0}, \ldots, \alpha_{n-1}\right\}$. We only present the case $Z_{0} \neq \emptyset$. Define $X^{\prime}=\{\zeta \in$ Ord For all $k<\omega, \exists p=\left(s_{\alpha}, A_{\alpha}\right) \in \mathbb{P}\left[\left(Z_{0} \subseteq \operatorname{dom}(p)\right) \wedge\left(\operatorname{otp}\left(s_{\alpha_{0}}\right) \geq k\right) \wedge\left(p \nmid Z_{0} \in G \uparrow Z_{0}\right) \wedge(p \Vdash\right.$ $\left.\left.\left.\varphi\left(\check{\zeta}, \vec{\xi}, \dot{C}_{\alpha_{0}}, \ldots, \dot{C}_{\alpha_{n-1}}, \dot{D}\right)\right)\right]\right\} \in V\left[G \uparrow Z_{0}\right]$. We claim that $X=X^{\prime}$. To see this, if $\zeta \in X$, then there is $p=\left(s_{\alpha}, A_{\alpha}\right) \in G$ such that $p \Vdash \varphi\left(\check{\zeta}, \vec{\xi}, \dot{C}_{\alpha_{0}}, \ldots, \dot{C}_{\alpha_{n-1}}, \dot{D}\right)$. By density, it is possible to assume that $Z_{0} \subseteq \operatorname{dom}(p)$. Since $G$ contains conditions where the $\alpha_{0}$-th stem is of arbitrary finite order type, it is possible also to arrange that $\operatorname{otp}\left(s_{\alpha_{0}}\right) \geq k$ for any given $k<\omega$. Hence, $\zeta \in X^{\prime}$.

Conversely, assume that $\zeta \notin X$. Take $q=\left(t_{\alpha}, B_{\alpha}\right) \in G$ to be such that $q \Vdash \neg \varphi\left(\check{\zeta}, \vec{\xi}, \dot{C}_{\alpha_{0}}, \ldots, \dot{C}_{\alpha_{n-1}}, \dot{D}\right)$. We may assume that $Z_{0} \subseteq \operatorname{dom}(q)$. Suppose that there were $p=\left(s_{\alpha}, A_{\alpha}\right) \in \mathbb{P}$ such that

$$
\left(Z_{0} \subseteq \operatorname{dom}(p)\right) \wedge\left(\operatorname{otp}\left(s_{\alpha_{0}}\right) \geq \operatorname{otp}\left(t_{\alpha_{0}}\right)\right) \wedge\left(p \uparrow Z_{0} \in G \uparrow Z_{0}\right) \wedge\left(p \Vdash \varphi\left(\check{\zeta}, \vec{\xi}, \dot{C}_{\alpha_{0}}, \ldots, \dot{C}_{\alpha_{n-1}}, \dot{D}\right)\right)
$$


Take some $p^{\prime}=\left(s_{\alpha}^{\prime}, A_{\alpha}^{\prime}\right) \in G$ such that $p^{\prime} \uparrow Z_{0}=p\left\lceil Z_{0}\right.$. Since $G$ is a generic filter, there is $p^{\prime \prime}=\left(s_{\alpha}^{\prime \prime}, A_{\alpha}^{\prime \prime}\right) \in G$ such that $\left(s_{a}^{\prime \prime}, A_{\alpha}^{\prime \prime}\right) \leq\left(t_{\alpha}, B_{\alpha}\right)$ and $\left(s_{a}^{\prime \prime}, A_{\alpha}^{\prime \prime}\right) \leq\left(s_{\alpha}^{\prime}, A_{\alpha}^{\prime}\right)$. Then $t_{\alpha_{0}}$ and $s_{\alpha_{0}}^{\prime}$ are both initial segments of $s_{\alpha_{0}}^{\prime \prime}$, and $t_{\alpha_{0}}$ is an initial segment of $s_{\alpha_{0}}^{\prime}=s_{\alpha_{0}}$. Let $\ell=\operatorname{otp}\left(s_{\alpha_{0}} \backslash t_{\alpha_{0}}\right)$. Define a condition $q^{*}=\left(s_{\alpha}^{*}, A_{\alpha}^{\prime \prime}\right) \leq\left(t_{\alpha}, B_{\alpha}\right)=q$ by

$$
s_{\alpha}^{*}=\left\{\begin{array}{l}
t_{\alpha} \cup\left\{\left(s_{\alpha}^{\prime \prime} \backslash t_{\alpha}\right)[i] \mid i<\ell\right\} \text { if } \alpha \in \operatorname{dom}(q), \\
\emptyset \text { otherwise }
\end{array}\right.
$$

where $\left(s_{\alpha}^{\prime \prime} \backslash t_{\alpha}\right)[i]$ is the $i$-th element of the monotone enumeration of $s_{\alpha}^{\prime \prime} \backslash t_{\alpha}$. Note that for $\alpha \in \operatorname{dom}(q), s_{\alpha}^{*}$ may be a proper initial segment of $s_{\alpha}^{\prime \prime}$. Since $p^{\prime \prime}=\left(s_{\alpha}^{\prime \prime}, A_{\alpha}^{\prime \prime}\right) \in G, p^{\prime}=\left(s_{\alpha}^{\prime}, A_{\alpha}^{\prime}\right) \in G$, $p^{\prime \prime} \leq p^{\prime}, p^{\prime} \uparrow Z_{0}=p\left\lceil Z_{0}\right.$, and $G$ is a generic filter, the conditions $q^{*}$ and $p$ have the same stems on the support $Z_{0}$. By thinning out reservoir sets, we can further assume that $q^{*} \uparrow Z_{0}=p \nmid Z_{0}$. But then, $q^{*} \Vdash \neg \varphi\left(\check{\zeta}, \vec{\xi}, \dot{C}_{\alpha_{0}}, \ldots, \dot{C}_{\alpha_{n-1}}, \dot{D}\right)$ and $p \Vdash \varphi\left(\check{\zeta}, \vec{\xi}, \dot{C}_{\alpha_{0}}, \ldots, \dot{C}_{\alpha_{n-1}}, \dot{D}\right)$. However, this contradicts Lemma 7. Thus, there are no $p$ satisfying (2), and hence $\zeta \notin X^{\prime}$.

\section{Applications to the Singular Cardinals Hypothesis}

Theorem 5 Assume that $V \vDash " Z F C+\kappa$ is a measurable cardinal". Then there is a partial ordering $\mathbb{P} \in V$ and a symmetric submodel $N \subseteq V^{\mathbb{P}}$ in which there is a surjective failure of the Singular Cardinals Hypothesis at $\kappa$. In particular, for $\beta \geq 2$, there is such a model $N$ with a surjection from $[\kappa]^{\omega}$ onto $\kappa^{+\beta}$ in $N$, and $N \vDash " G C H$ holds below $\kappa "$.

Proof Without loss of generality, by forcing or using an appropriate constructible inner model, we may also assume that $V \vDash G C H$. Define the forcing $(\mathbb{P}, \leq)=\left(\mathbb{P}_{Z}, \leq\right)$ with $Z=\kappa^{+\beta}$ as above. Let $V[G]$ be a generic extension of $V$ by $\mathbb{P}$, and let $\left(C_{\alpha}\right)_{\alpha<\kappa^{+\beta}}$ be the sequence of Prikry sequences adjoined by $G$. Then form the model

$$
N=\operatorname{HOD}^{V[G]}\left(\left\{C_{\alpha} \mid \alpha<\kappa^{+\beta}\right\} \cup\left\{\left(\tilde{C}_{\alpha} \mid \alpha<\kappa^{+\beta}\right)\right\}\right) .
$$

Every set of ordinals in $N$ is of the form

$$
X=\left\{\zeta \in \operatorname{Ord} \mid V[G] \vDash \varphi\left(\zeta, \vec{\xi}, C_{\alpha_{0}}, \ldots, C_{\alpha_{n-1}},\left(\tilde{C}_{\alpha} \mid \alpha<\kappa^{+\beta}\right)\right)\right\}
$$


for some $\in$-formula $\varphi, \vec{\xi} \in$ Ord, and $\alpha_{0}, \ldots, \alpha_{n-1}<\kappa^{+\beta}$. By Lemma 8 ,

$$
X \in V\left[G \uparrow\left\{\alpha_{0}, \ldots, \alpha_{n-1}\right\}\right]
$$

By Lemma 4, finite support parallel Prikry forcings do not add bounded subsets of $\kappa$. Hence, $\kappa$ is a singular cardinal in $N$, and $N \vDash$ "GCH holds below $\kappa$ ". By Lemma 3, there is a surjection $f:[\kappa]^{\omega} \rightarrow\left(\kappa^{+\beta}\right)^{V}$ in $N$. By Lemma 8 and Lemma $2,\left(\kappa^{+\beta}\right)^{V}=\left(\kappa^{+\beta}\right)^{N}$. Therefore, $f$ is a choiceless, surjective failure of $\mathrm{SCH}$.

Since by Lemma 8 and Lemma $4, N$ and $V$ contain the same bounded subsets of $\kappa,\left(V_{\kappa}\right)^{V}=$ $\left(V_{\kappa}\right)^{N}$. From this, it is possible to infer that any $x \in\left(V_{\kappa}\right)^{N}$ is well-orderable. Further, Lemma 8 and Lemma 4, together with the fact that in $N, \operatorname{cof}(\kappa)=\omega$, tell us that in $N$, there is a sequence of inaccessible cardinals $\left\langle\kappa_{i} \mid i<\omega\right\rangle$ whose limit is $\kappa$. These observations will be used in the construction of the witnessing model for Theorem 1 to be given in Section 6 .

\section{Collapsing cardinals}

We briefly describe the general method we shall use for symmetrically collapsing a singular cardinal $\kappa$ which is a limit of inaccessible cardinals down to small cardinals like $\aleph_{\omega}, \aleph_{\omega_{1}}$, or $\aleph_{\omega_{2}}$. Most of what we are about to discuss is found in [1, Section 4, pages $730-732]$, whose presentation we closely follow. In particular, our construction will result in a choiceless, symmetric inner model of a generic extension $V[G]$.

Assume that $\lambda$ represents in our ground model $V$ one of the cardinals $\omega, \omega_{1}$, or $\omega_{2}$. Let $V \vDash$ "ZF $+\left\langle\kappa_{i} \mid i<\lambda\right\rangle$ is a sequence of inaccessible cardinals whose limit is $\kappa "$. Note that it may or may not be the case that $\mathrm{AC}$ is true in $V$. Even if $\mathrm{AC}$ is false in $V$, by our remarks above, it will be possible to assume that $V_{\kappa}$ is well-orderable. Thus, " $\kappa_{i}$ is an inaccessible cardinal" will have the same meaning as when $\mathrm{AC}$ is true.

Take $\left\langle\lambda_{i} \mid i<\lambda\right\rangle$ as the sequence $\left\langle\kappa_{i} \mid i<\lambda\right\rangle$, together with its limit points less than $\kappa$. (If $\lambda=\omega$, then $\left\langle\lambda_{i} \mid i<\lambda\right\rangle=\left\langle\kappa_{i} \mid i<\lambda\right\rangle$.) Let $I=\{i<\lambda \mid i$ is either a successor ordinal or 0$\}$. For $i \in I$, let $\mathbb{P}_{i}=\operatorname{Coll}\left(\lambda_{i-1}^{+},<\lambda_{i}\right)$. Note that we take $\lambda_{-1}^{+}=\lambda^{+m}$ for some fixed $1 \leq m<\omega$, and that 
$\operatorname{Coll}\left(\lambda_{i-1}^{+},<\lambda_{i}\right)$ is the Lévy collapse of all cardinals in the open interval $\left(\lambda_{i-1}^{+}, \lambda_{i}\right)$ down to $\lambda_{i-1}^{+}$. We then define $\mathbb{P}=\prod_{i<\lambda} \mathbb{P}_{i}$ with full support.

Let $G$ be $\mathbb{P}$-generic over $V$, and for $i \in I$, let $G_{i}$ be the projection of $G$ onto $\mathbb{P}_{i}$. For $j \in I$, let $\mathbb{Q}_{j}=\prod_{i \leq j, i \in I} \mathbb{P}_{i}$ and $H_{j}=\prod_{i \leq j, i \in I} G_{i}$. It is the case, by the properties of the Lévy collapse and the Product Lemma, that $H_{j}$ is $\mathbb{Q}_{j}$-generic over $V$. Our symmetric inner model $N \subseteq V[G]$ can now be intuitively described as the least model of ZF extending $V$ which contains, for every $j \in I$, the set $H_{j}$.

In order to define $N$ more formally, we let $\mathcal{L}_{1}$ be the ramified sublanguage of the forcing language $\mathcal{L}$ with respect to $\mathbb{P}$ which contains symbols $\check{v}$ for each $v \in V$, a unary predicate symbol $\check{V}$ (to be interpreted $\check{V}(\check{v}) \Longleftrightarrow v \in V$ ), and symbols $\dot{H}_{j}$ for every $j \in I$. $N$ is then defined as follows.

$$
\begin{aligned}
& N_{0}=\emptyset . \\
& N_{\lambda}=\bigcup_{\alpha<\lambda} N_{\alpha} \text { if } \lambda \text { is a limit ordinal. } \\
& N_{\alpha+1}=\left\{\begin{array}{l|l}
x \subseteq N_{\alpha} & \begin{array}{l}
x \text { is definable over the model }\left\langle N_{\alpha}, \in, c\right\rangle_{c \in N_{\alpha}} \\
\text { via a term } \tau \in \mathcal{L}_{1} \text { of rank } \leq \alpha
\end{array}
\end{array}\right\} . \\
& N=\bigcup_{\alpha \in \operatorname{Ord}^{V}} N_{\alpha} \text {. }
\end{aligned}
$$

The relevant arguments found on [1, page 732] remain valid, regardless if GCH is true in $V$, since we can always assume that $V_{\kappa}$ is well-orderable and has cardinality $\kappa$. Therefore, it will be the case that $\kappa$ becomes in $N$ either $\aleph_{\omega}, \aleph_{\omega_{1}}$, or $\aleph_{\omega_{2}}$ (depending on whether initially, $\lambda=\omega, \lambda=\omega_{1}$, or $\left.\lambda=\omega_{2}\right)$. Since we will always be able to assume that $V \vDash$ "GCH holds below $\aleph_{\omega}$ ", $N \vDash$ "GCH holds below $\kappa "$. Further, as in [1], if $x \in N$ is a set of ordinals, then $x \in V\left[H_{j}\right]$ for some $j<\lambda$. Hence, since $\mathbb{Q}_{j} \in V_{\kappa}$, which means that $\mathbb{Q}_{j}$ is well-orderable, the cardinal and cofinality structure in $N$ above $\kappa$ is the same as in $V$. In addition, standard arguments (see [1]) show that either $N \vDash \neg \mathrm{AC}_{\omega}, N \vDash \mathrm{DC}$, or $N \vDash \mathrm{DC}_{\omega_{1}}$, depending if $\lambda=\omega, \lambda=\omega_{1}$, or $\lambda=\omega_{2}$ respectively.

\section{$6 \quad$ Upper bounds}

In this section, we prove the forcing direction for Theorems $1-4$. This will establish the upper bounds in consistency strength for each of these theorems. We will present a uniform proof 
throughout. Our general paradigm will be to force over the appropriate model $V^{\prime}$, which is obtained from our ground model $V$, using the collapses described in Section 5 . We then build the choiceless inner model $N$ described in Section 5, which will end up being our witnessing model.

For Theorem 1, we let $V \vDash$ "ZFC $+\mathrm{GCH}+\kappa$ is a measurable cardinal". Fix an arbitrary $\alpha \geq 2$. By Theorem 5 and the discussion found in Section 4, we may assume that there is a partial ordering $\mathbb{P} \in V$ and a choiceless, symmetric submodel $V^{\prime} \subseteq V^{\mathbb{P}}$ such that $V^{\prime} \vDash$ "GCH holds below $\kappa+\operatorname{cof}(\kappa)=\omega+$ There is a surjection $f:[\kappa]^{\omega} \rightarrow \kappa^{+\alpha}+V_{\kappa}$ is well-orderable + There is a sequence of inaccessible cardinals $\left\langle\kappa_{i} \mid i<\omega\right\rangle$ whose limit is $\kappa "$.

For Theorem 2, let $n<\omega, n \geq 2$ be fixed, and suppose that $V \vDash " \mathrm{ZFC}+\exists \kappa[(\operatorname{cof}(\kappa)=$ $\left.\omega) \wedge(\forall i<\omega)(\forall \lambda<\kappa)(\exists \delta<\kappa)\left[(\delta>\lambda) \wedge\left(o(\delta) \geq \delta^{+i}\right)\right]\right]$ ". Once again, by passing to the appropriate inner model if necessary, we may assume that $V \vDash \mathrm{GCH}$ as well. By the work of [7] (see also [6]), we may assume that $V$ has been generically extended to a model $V^{\prime}$ such that $V^{\prime} \vDash$ ' $\mathrm{ZFC}+\mathrm{GCH}$ holds below $\kappa+2^{\kappa}=\kappa^{+\omega_{n}}$, and there is an injective $f: \kappa^{+\omega_{n}} \rightarrow[\kappa]^{\omega}+$ There is a sequence of inaccessible cardinals $\left\langle\kappa_{i} \mid i<\omega\right\rangle$ whose limit is $\kappa "$.

For Theorems 3 and 4, suppose that $V \vDash " \mathrm{ZFC}+\exists \kappa\left[o(\kappa)=\kappa^{++}+\zeta\right]$ ". Here, $\zeta=\omega_{2}$ for Theorem 3, and $\zeta=\omega_{1}$ for Theorem 4. As above, by passing to the appropriate inner model if necessary, we may assume that $V \vDash \mathrm{GCH}$ as well. By the work of [16] and [15] (see also the remark immediately following the statement of Theorem 1 on [12, page 274]), by first forcing to make $2^{\kappa}=\kappa^{++}$while preserving that $o(\kappa)=\zeta$, and then forcing to change $\kappa^{\prime}$ 's cofinality to $\zeta$, we may assume that $V$ has been generically extended to a model $V^{\prime}$ such that $V^{\prime} \vDash$ "ZFC + GCH holds below $\kappa+\operatorname{cof}(\kappa)=\zeta+2^{\kappa}=\kappa^{++}$, and there is an injective $f: \kappa^{++} \rightarrow[\kappa]^{\zeta}+$ There is a sequence of inaccessible cardinals $\left\langle\kappa_{i} \mid i<\zeta\right\rangle$ whose limit is $\kappa^{\prime \prime}$.

We may now collapse over every $V^{\prime}$ as in Section 5 and build a choiceless, symmetric submodel $N$ of the generic extension via the collapses. For Theorem 2 , we take $m=n$, where $m$ is as mentioned in Section 5. For Theorems 1, 3, and 4, the value of $m$ is irrelevant. In each instance, $N$ witnesses the forcing direction of either Theorem 1, Theorem 2, Theorem 3, or Theorem 4. 


\section{Lower bounds and the proofs of Theorems $1-4$}

In this section, we prove the inner model portions of Theorems $1-4$, i.e., we obtain lower bounds in consistency strength for each of these theorems. Once this has been done, the proofs of Theorems $1-4$ will be complete.

We begin by establishing the lower bound in consistency strength for Theorem 1 .

Theorem 6 Assume that SCH fails in a surjective way in a model $V$ of $Z F$. Then there is an inner model of ZFC with a measurable cardinal.

Proof Let $\kappa$ be a singular cardinal such that $(\forall \nu<\kappa)\left[2^{\nu}<\kappa\right]$, and let $f:[\kappa]^{\operatorname{cof}(\kappa)} \rightarrow \kappa^{++}$be a surjection. Let $\lambda=\operatorname{cof}(\kappa)+\aleph_{2}$. Then because $\kappa$ is a limit cardinal, $\lambda<\kappa$. Assume towards a contradiction that there is no inner model of ZFC with a measurable cardinal. Let $K$ be (the canonical term for) the Dodd-Jensen core model $K$ (see [3] and [4] for further details) below a measurable cardinal. For $Y \subseteq$ Ord, take $g_{Y}$ : otp $(Y) \leftrightarrow Y$ to be the uniquely defined order preserving map between otp $(Y)$ and $Y$.

Consider $X \in[\kappa]^{\operatorname{cof}(\kappa)}$. Let $\operatorname{HOD}[X]$ be the smallest inner model such that $\operatorname{HOD} \cup\{X\} \subseteq$ $\operatorname{HOD}[X]$. By $[1$, Proposition $1.1(1)], \operatorname{HOD}[X]$ is a set generic extension of HOD, so $\operatorname{HOD}[X] \subseteq V$ is a model of ZFC. Further, in $\operatorname{HOD}[X]$, there is no inner model of ZFC with a measurable cardinal. By the Dodd-Jensen covering theorem in $\operatorname{HOD}[X]$, there is a covering set $Y \in K^{\mathrm{HOD}[X]}$, $X \subseteq Y \subseteq \kappa, \operatorname{otp}(Y)<\lambda$. Let $Z=g_{Y}^{-1}[X] \in \wp(\lambda)$. Then

$$
X=g_{Y}[Z] \text { for some } Y \in \wp(\kappa) \cap K^{\mathrm{HOD}[X]} \text { and } Z \in \wp(\lambda) \text {. }
$$

By the absoluteness properties of the Dodd-Jensen core model, $K^{\mathrm{HOD}[X]}=K^{\mathrm{HOD}}$. Consequently,

$$
X=g_{Y}[Z] \text { for some } Y \in \wp(\kappa) \cap K^{\mathrm{HOD}} \text { and } Z \in \wp(\lambda) \text {. }
$$

Since GCH holds in $K^{\mathrm{HOD}}$, take a surjective $k: \kappa^{+} \rightarrow \wp(\kappa) \cap K^{\mathrm{HOD}}$. Since $2^{\lambda}<\kappa$, take a surjective $h: \kappa \rightarrow \wp(\lambda)$. By (4), the map

$$
(\gamma, \eta) \mapsto f\left(g_{k(\gamma)}[h(\eta)]\right)
$$


is a surjection from $\kappa^{+} \times \kappa$ onto $\kappa^{++}$. This contradiction completes the proof of Theorem 6 .

We establish the lower bounds in consistency strength for Theorems $2-4$ by using Gitik's work of [6] (see also [7]) and Gitik and Mitchell's work of [12]. For Theorem 2, we work from a choiceless model $N$ of ZF such that $N \vDash " G C H$ holds below $\aleph_{\omega}+$ There is an injective $f: \aleph_{\omega_{n}} \rightarrow\left[\aleph_{\omega}\right]^{\omega}$, where $1 \leq n<\omega$ ". In particular, we can let $x=\left\langle x_{\alpha} \mid \alpha<\left(\aleph_{\omega_{n}}\right)^{N}\right\rangle \in N$ be a sequence of distinct subsets of $\left(\aleph_{\omega}\right)^{N}$. Let $y \in N$ be a countable sequence of ordinals having order type $\omega$ cofinal in $\lambda=\left(\aleph_{\omega}\right)^{N}$. In $L[x, y] \vDash \mathrm{ZFC}, \lambda$ is a singular strong limit cardinal of cofinality $\omega$. Further, it is the case that $L[x, y] \vDash " 2^{\lambda} \geq \lambda^{+\omega_{n}} "$ (since $\left(\omega_{n}\right)^{N} \geq\left(\omega_{n}\right)^{L[x, y]}$ ). By [12, Theorem 1, clause 3] and the remark at the bottom of $\left[6\right.$, page 1], in the core model $(K)^{L[x, y]},\left\{\delta<\lambda \mid o(\delta) \geq \delta^{+i}\right\}$ is cofinal in $\lambda$ for each $i<\omega$. If $\operatorname{cof}(\lambda)=\omega$ in $(K)^{L[x, y]}$, then we are done. If not, then $\operatorname{cof}(\lambda) \geq \omega_{1}$ in $(K)^{L[x, y]}$. Work in the core model, and for each $i<\omega$, let $S_{i}=\left\{\delta<\lambda \mid o(\delta) \geq \delta^{+i}\right\}$. Define $\delta_{0}$ as the minimal member of $S_{0}$, and for $1 \leq i<\omega$, take $\delta_{i}$ as the minimal member of $S_{i}$ greater than $\delta_{i-1}$. Then $\zeta=\sup \left(\left\{\delta_{i} \mid i<\omega\right\}\right)<\lambda$ is such that $\operatorname{cof}(\zeta)=\omega$ and $\left\{\delta<\zeta \mid o(\delta) \geq \delta^{+i}\right\}$ is cofinal in $\zeta$ for each $i<\omega$. We have consequently established the existence of a model, i.e., $(K)^{L[x, y]}$, containing the cardinal $\kappa$ mentioned in the hypotheses for Theorem 2.

For Theorems 3 and 4b), we work from a choiceless model $N$ of ZF such that $N \vDash$ "GCH holds below $\aleph_{\zeta}+$ There is an injective $f: \aleph_{\zeta+2} \rightarrow\left[\aleph_{\zeta}\right]^{\zeta "}$. Here, $\zeta=\left(\omega_{2}\right)^{N}$ for Theorem 3 , and $\zeta=\left(\omega_{1}\right)^{N}$ for Theorem 4b). In particular, we can let $x=\left\langle x_{\alpha}\right| \alpha\left\langle\left(\aleph_{\zeta+2}\right)^{N}\right\rangle \in N$ be a sequence of distinct subsets of $\left(\aleph_{\zeta}\right)^{N}$. Let $y \in N$ be an uncountable sequence of ordinals having order type $\zeta$ cofinal in $\lambda=\left(\aleph_{\zeta}\right)^{N}$. In $L[x, y] \vDash \mathrm{ZFC}, \lambda$ is a singular strong limit cardinal of uncountable cofinality such that $2^{\lambda} \geq \lambda^{++}\left(\right.$since $\left.\left(\lambda^{++}\right)^{N} \geq\left(\lambda^{++}\right)^{L[x, y]}\right)$. As above, let $(K)^{L[x, y]}$ be the core model. By [12, Theorem 1, clause 1], when $\zeta=\left(\omega_{2}\right)^{N}$, since $L[x, y] \vDash " \lambda$ is singular and $\operatorname{cof}(\lambda)=\gamma \geq\left(\omega_{2}\right)^{N} \geq \omega_{2}$ ", $(K)^{L[x, y]} \vDash " o(\lambda) \geq \lambda^{++}+\gamma$ ". By [12, Theorem 1, clause 2], when $\zeta=\left(\omega_{1}\right)^{N}$, since $L[x, y] \vDash " \lambda$ is singular and $\operatorname{cof}(\lambda)=\gamma \geq\left(\omega_{1}\right)^{N} \geq \omega_{1} ",(K)^{L[x, y]} \vDash " o(\lambda) \geq \lambda^{++} "$. For either value of $\zeta$, we have once again established the existence of a model containing the cardinal $\kappa$ mentioned in the hypothesis or conclusion of Theorems 3 and $4 \mathrm{~b})$.

Theorem 6 and the discussion in the preceding two paragraphs establish the lower bounds in 
consistency strength for Theorems $1-4$, thereby completing the proofs of these theorems.

\section{Concluding remarks}

In conclusion, we would like to make several remarks concerning Theorems $1-4$. To begin, we observe that there is nothing special about $\aleph_{\omega}, \aleph_{\omega_{1}}$, and $\aleph_{\omega_{2}}$ in Theorems $1-4$. It is certainly possible to collapse the cardinal $\kappa$ in question to other singular limit cardinals, such as $\aleph_{\omega+\omega}, \aleph_{\aleph_{\omega}}$, $\aleph_{\omega_{5}}, \aleph_{\aleph_{\omega_{6}}}$, etc., and obtain similar equiconsistencies. We leave it to readers to work out the exact details for themselves.

We observe that the dichotomy between Theorems 3 and 4 comes from the fact that [12, Theorem 1] splits into the cases of a strong limit cardinal $\kappa$ of uncountable cofinality having cofinality at least $\omega_{2}$ and cofinality $\omega_{1}$. In the latter situation, we only know that in the core model, $o(\kappa)$ is greater than or equal to the size of the power set of $\kappa$. This does not appear to be a strong enough hypothesis in order to do the forcing necessary to construct the model containing the injection found in Theorem 4.

Our methods also make it possible to blow up the power sets of the singular limit cardinals in the injective failures of SCH given by Theorems $2-4$ arbitrarily high. For instance, starting with, e.g., a model for GCH containing a cardinal $\kappa$ which is $\lambda$ strong, where $\lambda>\kappa$ is inaccessible, it is possible (see [8]) to force $2^{\kappa}=\lambda$ while simultaneously changing $\kappa$ 's cofinality to $\omega$. Using a similar assumption, e.g., a model for $\mathrm{GCH}$ with a cardinal $\kappa$ such that $\lambda>\kappa$ is inaccessible and $o(\kappa)=\lambda+\omega_{1}$ or $o(\kappa)=\lambda+\omega_{2}$, it is possible to force $2^{\kappa}=\lambda$ and then change $\kappa$ 's cofinality to $\omega_{1}$ or

$\omega_{2}$. One may then symmetrically collapse $\kappa$ as in Theorems $2-4$ to be $\aleph_{\omega}, \aleph_{\omega_{1}}, \aleph_{\omega_{2}}$, etc., thereby producing a choiceless injective failure of $\mathrm{SCH}$ in which there is an injection from a regular limit cardinal into $\wp\left(\aleph_{\omega}\right), \wp\left(\aleph_{\omega_{1}}\right), \wp\left(\aleph_{\omega_{2}}\right)$, etc.

However, under the circumstances just described, it may not be possible to obtain an equiconsistency. For instance, assume that an injection from a regular limit cardinal into $\wp\left(\aleph_{\omega}\right)$ is obtained. It does not yet seem to be possible to force the existence of the cardinal used in our construction, i.e., a singular strong limit cardinal $\delta$ of cofinality $\omega$ such that $2^{\delta}$ is a regular limit cardinal, starting 
with an inaccessible cardinal above the cardinal $\kappa$ mentioned in the hypotheses of Theorem 2. (See [7, Section 4] for the largest possible value to which the size of the power set of a cardinal $\kappa$ as in the hypotheses of Theorem 2 can currently be blown up.) By [12, Theorem 1, clause 3] and the remark at the bottom of [6, page 1], $\delta$ will satisfy the properties of the cardinal of the hypotheses of Theorem 2 in the core model. Of course, because of the dichotomy described above, an equiconsistency also does not appear to be possible when forcing an injection from a regular limit cardinal into $\wp\left(\aleph_{\omega_{1}}\right)$, although there is no problem in obtaining an equiconsistency when forcing an injection from a regular limit cardinal into $\wp\left(\aleph_{\omega_{2}}\right), \wp\left(\aleph_{\omega_{3}}\right)$, etc.

As we noted earlier, we have not been able to replace in Theorem 1 the surjection from $\left[\aleph_{\omega}\right]^{\omega}$ onto $\aleph_{\omega+2}$ with a surjection from $\wp\left(\aleph_{\omega}\right)$ onto $\aleph_{\omega+2}$. The reason is that the proof of Theorem 6

looks as though it requires the former sort of surjection. We therefore ask if the equiconsistency of Theorem 1 can be obtained using only the latter kind of surjection.

Finally, we ask if it is possible to prove analogues of Theorem 1 for singular strong limit cardinals of uncountable cofinality. Magidor's forcing of [15] for changing to an uncountable cofinality does not appear to be amenable to the analyses found in Sections $2-4$, which seems to pose a formidable barrier.

\section{References}

[1] A. Apter, P. Koepke, "The Consistency Strength of $\aleph_{\omega}$ and $\aleph_{\omega_{1}}$ being Rowbottom Cardinals without the Axiom of Choice", Archive for Mathematical Logic 45, 2006, 721-737.

[2] K. Devlin, R. B. Jensen, "Marginalia to a Theorem of Silver", in: ISILC Logic Conference (Proceedings of the International Summer Institute and Logic Colloquium, Kiel, 1974), Lecture Notes in Mathematics 499, Springer-Verlag, Berlin and New York, 1975, 115-142.

[3] A. J. Dodd, The Core Model, London Mathematical Society Lecture Note Series \#61, Cambridge University Press, Cambridge, 1982.

[4] A. J. Dodd, R. B. Jensen, "The Core Model", Annals of Mathematical Logic 20, 1981, 43-75. 
[5] W. Easton, "Powers of Regular Cardinals", Annals of Mathematical Logic 1, 1970, 139-178.

[6] M. Gitik, "Blowing up Power of a Singular Cardinal - Wider Gaps", Annals of Pure and Applied Logic 116, 2002, 1-38.

[7] M. Gitik, "Dropping Cofinalities and Gaps", preprint.

[8] M. Gitik, "Prikry-type Forcings", forthcoming article in the Handbook of Set Theory.

[9] M. Gitik, "The Negation of the Singular Cardinal Hypothesis from $o(\kappa)=\kappa^{++", ~ A n n a l s ~ o f ~}$ Pure and Applied Logic 43, 1989, 209-234.

[10] M. Gitik, "The Power Set Function", in: Proceedings of the International Congress of Mathematicians (Beijing 2002), volume 1, Higher Education Press, Beijing, 2002, 507-513.

[11] M. Gitik, "The Strength of the Failure of the Singular Cardinal Hypothesis", Annals of Pure and Applied Logic 51, 1991, 215-240.

[12] M. Gitik, W. Mitchell, "Indiscernible Sequences for Extenders, and the Singular Cardinal Hypothesis", Annals of Pure and Applied Logic 82, 1996, 273-316.

[13] M. Gitik, R. Schindler, S. Shelah, "Pcf Theory and Woodin Cardinals", in: Logic Colloquium '02, Lecture Notes in Logic 27, Association for Symbolic Logic, La Jolla, California, 2006, $172-205$.

[14] T. Jech, Set Theory: The Third Millennium Edition, Revised and Expanded, Springer-Verlag, Berlin and New York, 2003.

[15] M. Magidor, "Changing Cofinality of Cardinals", Fundamenta Mathematicae 99, 1978, 61-71.

[16] M. McDermott, Doctoral Dissertation, Oxford University.

[17] S. Shelah, Cardinal Arithmetic, Oxford Logic Guides 29, Oxford University Press, New York, 1994. 
[18] J. Silver, "On the Singular Cardinals Problem", in: Proceedings of the International Congress of Mathematicians (Vancouver, British Columbia 1974), volume 1, Canadian Mathematical Congress, Montreal, Quebec, 1975, 265-268. 